\title{
Bayesian Analysis for Extracting Properties of the Nuclear Equation of State from Observational Data Including Tidal Deformability from GW170817 ${ }^{\dagger}$
}

\author{
Alexander Ayriyan 1(D), David Alvarez-Castillo ${ }^{2}(\mathbb{D})$, David Blaschke ${ }^{2,3,4, * \mathbb{D}}$ and \\ Hovik Grigorian 1,5 \\ 1 Laboratory for Information Technologies, Joint Institute for Nuclear Research, Joliot-Curie Street 6, \\ Dubna 141980, Russia; ayriyan@jinr.ru (A.A.); hovikgrigorian@gmail.com (H.G.) \\ 2 Bogoliubov Laboratory for Theoretical Physics, Joint Institute for Nuclear Research, Joliot-Curie Street 6, \\ Dubna 141980, Russia; sculkaputz@gmail.com \\ 3 Institute of Theoretical Physics, University of Wroclaw, Max Born Place 9, 50-204 Wroclaw, Poland \\ 4 National Research Nuclear University (MEPhI), Kashirskoe Shosse 31, Moscow 115409, Russia \\ 5 Department of Physics, Yerevan State University, Alek Manukyan Str. 1, Yerevan 0025, Armenia \\ * Correspondence: david.blaschke@gmail.com \\ $+\quad$ This paper is based on the talk at the 7th International Conference on New Frontiers in Physics \\ (ICNFP 2018), Crete, Greece, 4-12 July 2018.
}

Received: 27 December 2018; Accepted: 5 February 2019; Published: 14 February 2019

\begin{abstract}
We develop a Bayesian analysis method for selecting the most probable equation of state under a set of constraints from compact star physics, which now include the tidal deformability from GW170817. We apply this method for the first time to a two-parameter family of hybrid equations of state that is based on realistic models for the hadronic phase (KVORcut02) and the quark matter phase $(\mathrm{SFM} \alpha)$ which produce a third family of hybrid stars in the mass-radius diagram. One parameter $(\alpha)$ characterizes the screening of the string tension in the string-flip model of quark matter while the other $\left(\Delta_{P}\right)$ belongs to the mixed phase construction that mimics the thermodynamics of pasta phases and includes the Maxwell construction as a limiting case for $\Delta_{P}=0$. We present the corresponding results for compact star properties like mass, radius and tidal deformabilities and use empirical data for them in the newly developed Bayesian analysis method to obtain the probabilities for the model parameters within their considered range.
\end{abstract}

Keywords: bayesian analysis; quark-hadron phase transition; pasta phases; hybrid compact stars; mass-radius relation; GW170817; tidal deformability

\section{Introduction}

The determination of the equation of state (EoS) of compact star (CS) interiors is a subject of active research by means of both laboratory measurements and astronomical observations. One of the most urgent questions concerns the possible existence of deconfined quark matter in CS cores, where matter densities can exceed by several times the nuclear saturation value, $n_{0}=0.15 \mathrm{fm}^{-3}$, the typical density in large atomic nuclei. Hybrid compact stars have an inner core composed of quark matter surrounded by an outer core of nuclear matter. Of particular interest is the CS mass twin (MT) phenomenon [1]: when a pair of stars has the same gravitational mass but different radii, with the larger star being composed only of hadronic matter and the smaller one being a hybrid star with a quark matter core. The presence of MTs is synonymous with the existence of a third family [2] of CS in the mass-radius $(M-R)$ diagram. It requires the CS EoS to feature a strong first order phase transition from hadron to quark matter [3-8], where the latent heat of the transition fulfills the Seidov criterion [9]. 
Should it turn out that the mass twin phenomenon can be discovered in CS observations, this would give indirect evidence for the existence of a critical endpoint in the phase diagram of quantum chromodynamics (QCD) $[4,5,10]$ which is sought for in relativistic heavy-ion collisions, so far without being conclusive. On the other hand, astronomical observations may provide measurements of masses and/or radii which are relevant for constraining the nuclear EoS via the one-to-one relationship provided by the Tolman-Oppenheimer-Volkoff (TOV) equations [11,12] which govern the general relativistic hydrodynamic stability of CS matter under its own gravity. Neutron star masses are accurately measured by monitoring binary pulsar dynamics, whereas the radii were determined so far with big uncertainties. The situation has changed with the first observation of the gravitational wave signal from the inspiral phase of the binary CS merger GW170817 [13], which allowed constraining the tidal deformability of both merging stars and thus their mass and radius [14-17].

Consequently, theoretical approaches are required to quantitatively assess the most probable EoS out of a whole class obtained by varying intrinsic model parameters. One of the most powerful methods is the Bayesian analysis (BA) or Bayesian interpretation of probability that allows for estimation of model parameters based on prior knowledge, in this case the already collected measurements. Several works in this direction include BA based on observations of X-ray bursters [18] that unfortunately suffer from uncertainties in the stellar atmosphere composition modeling required in the interpretation of the observed signal or the more general approach of [19] that includes a generic multipolytrope EoS with priors that include experimental nuclear matter pressure values and that is able to support the most massive CS of about $2 \mathrm{M}_{\odot}$ and reports an accuracy of about $30 \%$ in pressure values. The recent approach of [20] employs X-ray timing observations of accretion-powered millisecond pulsars, resulting in radius estimates of about $5 \%$ if the CS mass is known. Moreover, a more stringent analysis [21] that incorporates the hypothesis of the Direct Urca cooling constraint [22] in addition to the aforementioned measurements concludes that the neutron star radius has a value of $12.7 \pm 0.4 \mathrm{~km}$ for masses ranging from 1 up to $2 M_{\odot}$.

Despite the fact that the recent detection of gravitational radiation from the inspiral phase of the binary CS merger GW170817 has led to constraints on the tidal deformability of CS matter and to the discussion of the possibility that one or even both of the CS in the binary could be hybrid stars with quark matter interior and there would be a possibility to discover the mass twin phenomenon and thus a strong first order phase transition in CS matter [23-27], these data have not yet been included in BA studies such as the ones already listed above. Thus, it is of great interest to update such BA studies by incorporating this new information to constrain the CS EoS.

In this work, we consider the mass twin EoS and modifications to it in order to perform new Bayesian analysis calculations that, in addition to the previously used CS measurements in [28], will include the GW170817 data as priors to assess the probability of model parameters. Our model choice is the KVOR EoS for hadronic matter [29] together with the String-Flip approach [30] in its relativistic density functional formulation [31] for the deconfined quark matter regime.

\section{Hybrid EoS}

The hybrid EoS has been produced with the one-parameter replacement interpolation method (for a second order polynomial ansatz for $P(\mu)$ ) [32-34] which mimics the thermodynamic behavior of pasta phases in the coexistence region between the pure hadronic phase and the pure quark one.

The hadronic phase in this work is described by the well known KVOR equation of state [29] with a modification of stiffness as introduced in [35] and denoted as KVORcut2. This particular version of the KVOR EoS model is the stiffest one presented in that work and allows for fulfilling the condition [9] for the latent heat of phase transition to quark matter, so that the disconnected hybrid star family at higher densities is possible. 
The quark phase is based on the String-Flip Model with the so-called density functional [31] including the available volume fraction $\Phi$,

$$
\Phi\left(n_{\mathrm{B}}\right)=\mathrm{e}^{-\alpha n_{\mathrm{B}}^{2} \mathrm{fm}^{6}},
$$

where the parameter $\alpha$ describes the effective density-dependence of the confining interaction, and it is varied here in the range $\alpha \in[0.1, \ldots, 0.3]$. The value of this parameter is correlated with the critical density of the phase transition, as it is shown in the figures below. The larger $\alpha$, the lower the critical density and therefore the critical mass for the onset of the phase transition in the hybrid star. These systematics allows for calibrating the range of the model parameters with the observational data without contradicting the known properties of nuclear matter at saturation density.

For the construction of the hybrid star EoS from the hadronic and quark matter EoS, we employ here the mixed phase construction that is described in detail in Refs. [32-34]. It introduces the free parameter $\Delta_{P}=P\left(\mu_{c}\right) / P_{c}-1$, the pressure increment at the critical chemical potential $\mu_{c}$ relative to the critical pressure $P_{c}$ of the corresponding Maxwell construction, which is then obtained as a limiting case for $\Delta_{P}=0$. The resulting family of hybrid EoS corresponds to the one described in [32] (see Figure 1). This way of modelling the phase transition mimics the possibility of so-called pasta phases characterized by different geometric structures in the coexistence region of the transition sufficiently well, as has been shown in [36]. In that reference, it has also been demonstrated that the parameter $\Delta_{P}$ of the transition construction can be related to the value of the surface tension at the hadron to quark matter interface.
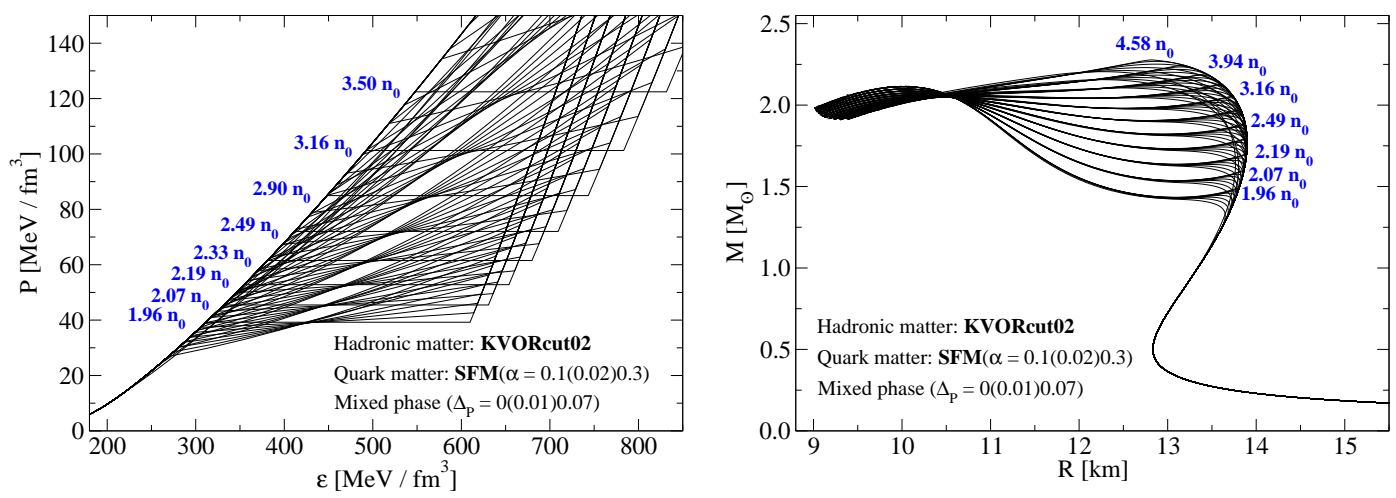

Figure 1. The set of hybrid EoS obtained by the mixed phase construction for different values of $\Delta_{P}$ and $\alpha$ is shown in the left panel, while in the right panel the corresponding set of compact star sequences in the mass-radius diagram is shown. The blue numbers show selected values of critical densities for the onset of the phase transitions obtained by a Maxwell construction.

\section{Neutron Star Configurations}

\subsection{Mass and Radius}

The structure and global properties of compact stars are obtained by solving the TOV equations,

$$
\begin{aligned}
\frac{d P(r)}{d r} & =-\frac{G M(r) \varepsilon(r)}{r^{2}} \frac{\left(1+\frac{P(r)}{\varepsilon(r)}\right)\left(1+\frac{4 \pi r^{3} P(r)}{M(r)}\right)}{\left(1-\frac{2 G M(r)}{r}\right)}, \\
\frac{d M(r)}{d r} & =4 \pi r^{2} \varepsilon(r),
\end{aligned}
$$


where $P(r), \varepsilon(r)$ and $M(r)$ are the profiles of pressure, energy density and enclosed mass as a function of the distance $r$ from the center of the star. The radius $R$ of the star is obtained from the condition of vanishing pressure at the surface $P(R)=0$ and the gravitational mass of the star is $M=M(R)$.

\subsection{Tidal Deformability}

The recent observation of gravitational waves from the inspiral phase of the binary CS merger GW170817 gives an estimation of the tidal deformabilities $\Lambda_{1}$ and $\Lambda_{2}$ of these stars; therefore, we also include this information in the analyses. The tidal deformability $\Lambda$ of a star can be calculated from the Einstein equation for small elliptic deformation as described in [37]. For the results of the calculation, see Figure 2.
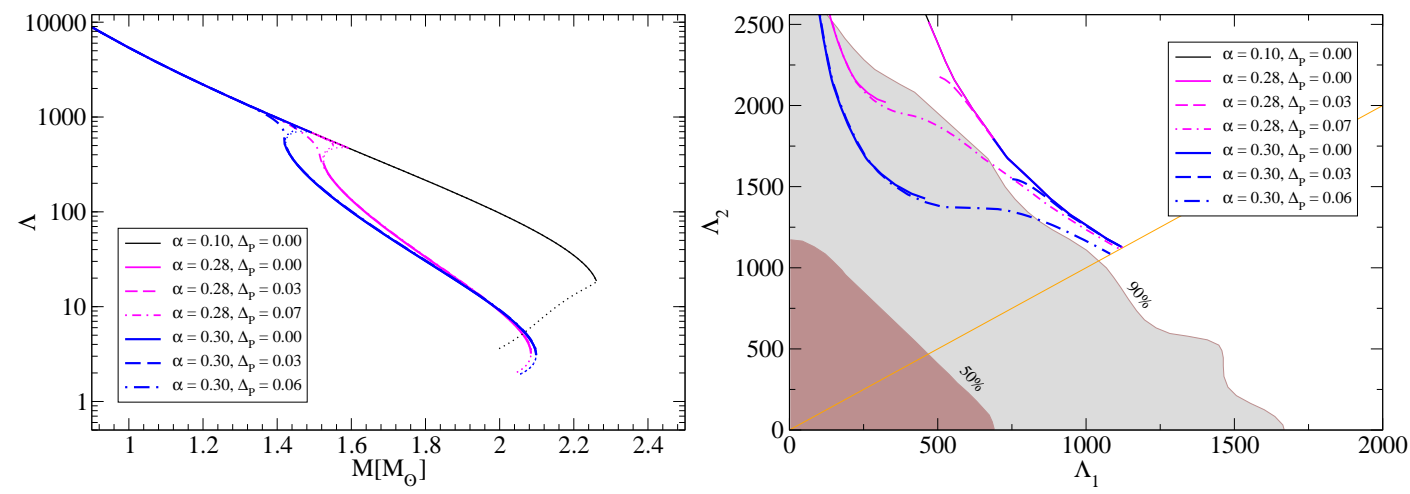

Figure 2. The dependence of the tidal deformability $\Lambda$ on the compact star mass (left panel) and the $\Lambda_{1}-\Lambda_{2}$ diagram for selected values of $\Delta_{P}$ and $\alpha$ (right panel). Comparison with the $90 \%$ confidence line of the LIGO-Virgo Collaboration for the low-spin prior of GW170817 [13] shows that, if the hadronic EoS is as stiff as DD2p40, at least one of the stars in the binary has to be a hybrid star in order to avoid a violation of the $\Lambda_{1}-\Lambda_{2}$ constraint. As can be seen from figure 17 of Ref. [23], the merger of two hybrid stars which is admissible when the onset mass for the deconfinement transition is lowered, e.g., by increasing $\alpha$, would lead to the appearance of a new branch in this diagram mimicking the pattern of a merger of two neutron stars with a rather soft nuclear EoS.

\section{Bayesian Inference for the EoS Models}

\subsection{Vector of Parameters}

The set of parameters of models could be represented in the parameter space with introduction of the vector of parameters, each vector is one fixed model from the types of hadronic described above, quark phases and transition construction:

$$
\vec{\pi}_{i}=\left\{\alpha_{(j)}, \Delta_{P(k)}\right\},
$$

where $i=0, \ldots, N-1$ and $i=N_{2} \times j+k$ and $j=0, \ldots, N_{1}-1, k=0, \ldots, N_{2}-1$ and $N_{1}$ and $N_{2}$ are number of values of model parameters $\alpha$ and $\Delta_{P}$ correspondingly.

\subsection{Likelihood of a Model for the $\Lambda_{1}-\Lambda_{2}$ Constraint from GW170817}

In order to implement the tidal deformability constraint on the compact stars EoS, reflected on the $\Lambda_{1}-\Lambda_{2}$ diagram that includes probability regions from GW170817 event [13,38], we employ the definition of the probability as an integral over the probability distribution function (PDF) $\beta\left(\Lambda_{1}, \Lambda_{2}\right)$ by

$$
P\left(E_{G W} \mid \pi_{i}\right)=\int_{l_{22}} \beta\left(\Lambda_{1}(\tau), \Lambda_{2}(\tau)\right) d \tau,
$$


when both stars in the binary merger belong to the second family branch of neutron stars (and $l_{22}$ is the corresponding path in the $\Lambda_{1}-\Lambda_{2}$ plane), or by

$$
P\left(E_{G W} \mid \pi_{i}\right)=\int_{l_{22}} \beta\left(\Lambda_{1}(\tau), \Lambda_{2}(\tau)\right) d \tau+\int_{l_{23}} \beta\left(\Lambda_{1}(\tau), \Lambda_{2}(\tau)\right) d \tau
$$

In case the parameter vector $\pi_{i}$ corresponds to a hybrid star equation of state with a third family of compact stars in the mass range relevant for the merger, then $l_{23}$ is the path in the $\Lambda_{1}-\Lambda_{2}$ plane corresponding to a binary merger composed of a neutron star and a hybrid star. The parameter $\tau$ defines the position of a point on the paths $l_{22}$ and $l_{23}$ in Equations (5) and (6). Note that the PDF has been reconstructed by the method Gaussian kernel density estimation with $\Lambda_{1}-\Lambda_{2}$ data given at LIGO web-page [39] (see Figure 3).
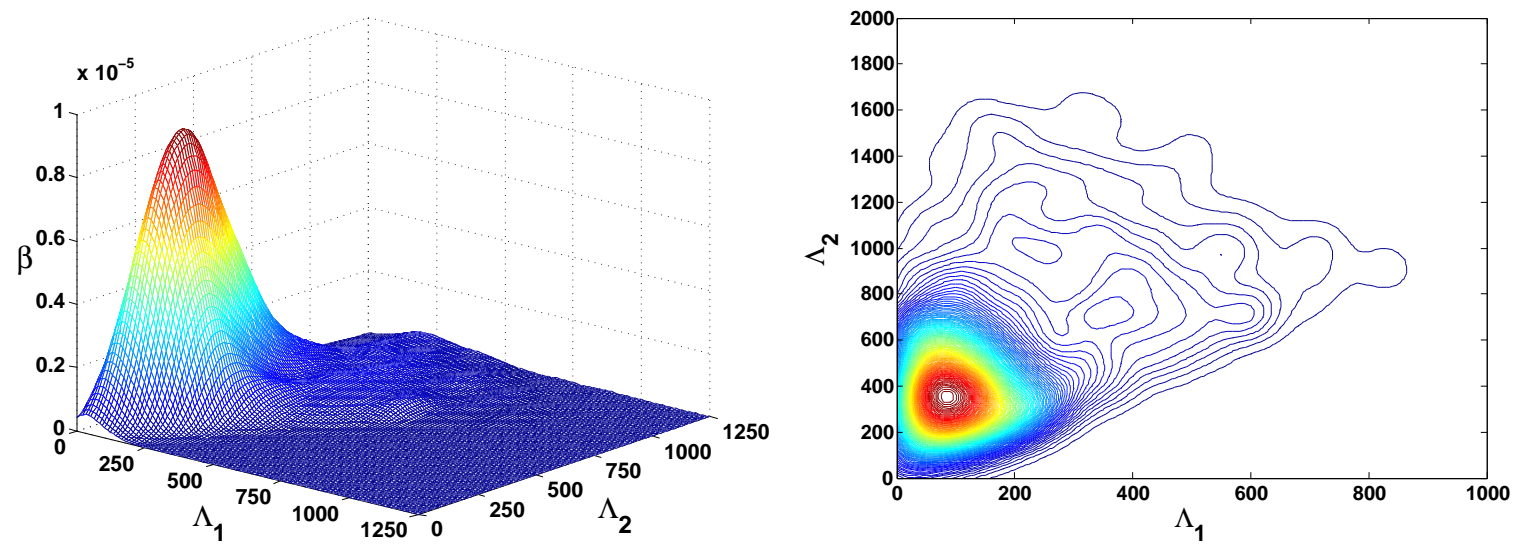

Figure 3. The PDF reconstructed with $\Lambda_{1}-\Lambda_{2}$ data for GW170817 from the LIGO website [39] as a 3D graphics (left panel) and as a contour plot (right panel).

\subsection{Likelihood of a Model for the Mass Constraint}

The likelihood of the model is the conditional probability of the expected value of the possible maximum mass for the given model parameter vector:

$$
P\left(E_{A} \mid \pi_{i}\right)=\Phi\left(M_{i}, \mu_{A}, \sigma_{A}\right),
$$

where $M_{i}$ is maximum mass of the given by $\pi_{i}$, and $\mu_{A}=2.01 \mathrm{M}_{\odot}$ and $\sigma_{A}=0.04 \mathrm{M}_{\odot}$ is the mass measurement [40].

\subsection{Posterior Distribution}

The full likelihood for the given $\pi_{i}$ can be calculated as a product of all likelihoods, since the considered constraints are independent from each other

$$
P\left(E \mid \vec{\pi}_{i}\right)=\prod_{m} P\left(E_{m} \mid \vec{\pi}_{i}\right) .
$$

The posterior distribution of models on the parameter diagram is given by Bayes' theorem

$$
P\left(\vec{\pi}_{i} \mid E\right)=\frac{P\left(E \mid \vec{\pi}_{i}\right) P\left(\vec{\pi}_{i}\right)}{\sum_{j=0}^{N-1} P\left(E \mid \vec{\pi}_{j}\right) P\left(\vec{\pi}_{j}\right)},
$$

where $P\left(\vec{\pi}_{j}\right)$ is a prior distribution of a model taken to be uniform: $P\left(\vec{\pi}_{j}\right)=1 / N$. 


\section{Results}

The results of the Bayesian analysis are given in Figure 4. The most likely EoS are those with a strong mixed phase effect described by $\Delta_{P}>0.04$ and with a large screening parameter $\alpha>0.28$, at the limit of the parameter range considered here. For these parameter sets, the phase transition and therefore the compactification of the hybrid star configuration occurs within the mass range that is relevant for GW170817. These results indicate that it should be worthwile to repeat this exploratory calculation with a wider set of parameters, in particular a larger screening parameter $\alpha$.
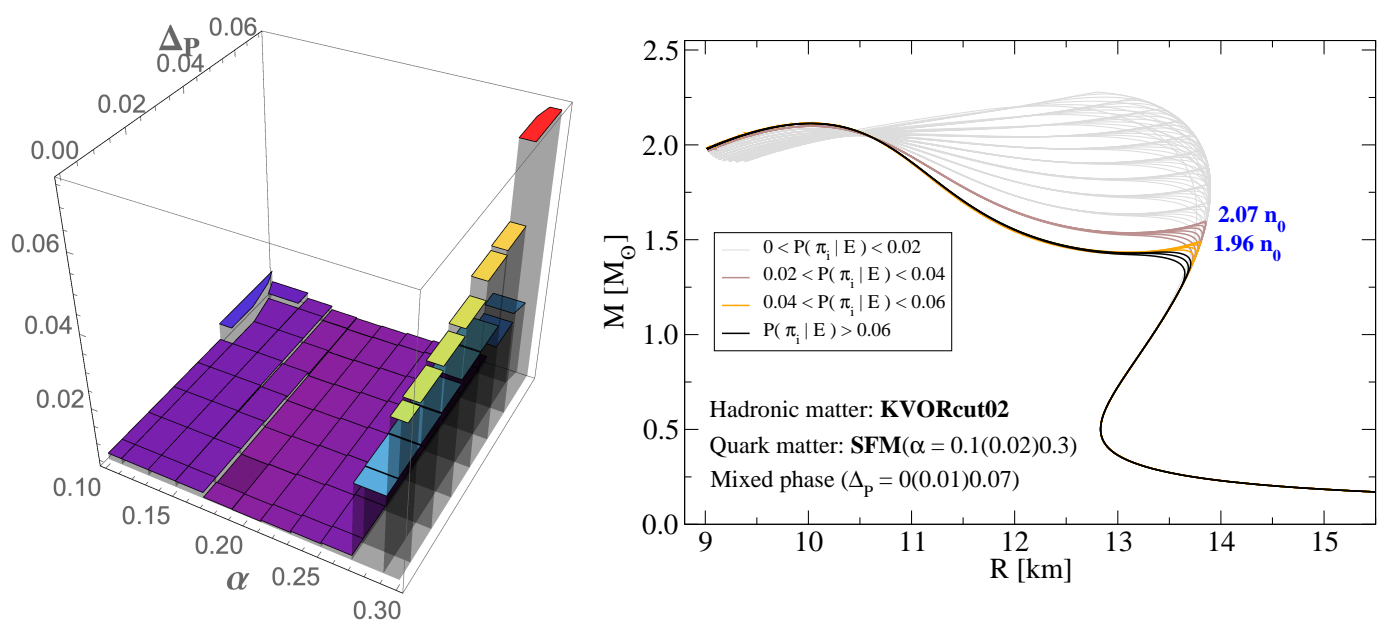

Figure 4. Left panel: The posterior distribution of models on the parameter space spanned by $\Delta_{P}$ and $\alpha$. Right panel: The compact star sequences in the mass-radius diagram labeled into four probability classes according to the results of the BA for the posterior distribution of the left panel. The grey, brown, orange and black lines show sequences for which the value of the posterior probability exceeds the thresholds of $0.0,0.02,0.04$ and 0.06 , respectively. Due to the restriction to the model and parameter range used in Ref. [32], the sequences with a lower onset mass for the deconfinement transition are not accessed for which an interpretation of GW170817 as a merger of two hybrid stars would be possible.

\section{Conclusions}

We have developed a Bayesian analysis method for selecting the most probable equation of state under a set of constraints from compact star physics, which now include the tidal deformability from GW170817. We have applied this method to a case study that employed the class of hybrid EoS introduced in [32] that allows for the existence of a third family of compact stars. We have investigated the requirements on future measurements to find the equivalent phenomenon of mass twins, i.e., two objects with the same mass but different, distinguishable radii. A mass-radius relation with two branches is mapped also on the $\Lambda_{1}-\Lambda_{2}$ diagram as it is shown here in Figure 3 and other recent publications [23-27]. Since binary compact star mergers are expected to be observed by the LIGO-Virgo Collaboration at a rate of 1-10 events per year, we expect that, with the observation of next merger events, a binodal structure of the PDF in the $\Lambda_{1}-\Lambda_{2}$ plane could become apparent as a manifestation of the low-mass twin case with an onset of the third family branch below $\sim 1.3 M_{\odot}$ if such a branch exists in nature. A similar suggestion has been already proposed by Christian et al. [25]. Such an observation would support the existence of an early phase transition, around $2 n_{0}$ for strong in-medium screening of the string tension.

Author Contributions: Conceptualization and methodology, A.A. and H.G.; software, A.A. and D.A.-C.; investigation, A.A.; data curation, A.A. and D.A.-C.; writing-original draft preparation, A.A. and D.A.-C.; writing-review and editing, D.B. and H.G.; visualization, A.A.; supervision, H.G.; project administration, D.B.; funding acquisition, D.B. and D.A.-C. 
Funding: A.A., D.B. and H.G. acknowledge support from the Russian Science Foundation under Grant No. 17-12-01427 for the work described in Sections 2-6. D.A.-C. is grateful for partial support from the Ter-Antonian-Smorodinsky program for collaboration between JINR and Armenian scientific institutions and from the Bogoliubov-Infeld program for collaboration between JINR and Polish Institutions.

Acknowledgments: We acknowledge discussions with Konstantin Maslov on the hybrid star EoS.

Conflicts of Interest: The authors declare no conflict of interest.

\section{References}

1. Glendenning, N.K.; Kettner, C. Nonidentical neutron star twins. Astron. Astrophys. 2000, 353, L9.

2. Gerlach, U.H. Equation of State at Supranuclear Densities and the Existence of a Third Family of Superdense Stars. Phys. Rev. 1968, 172, 1325-1330. [CrossRef]

3. Alford, M.G.; Han, S.; Prakash, M. Generic conditions for stable hybrid stars. Phys. Rev. D 2013, 88, 083013. [CrossRef]

4. Alvarez-Castillo, D.E.; Blaschke, D. Proving the CEP with compact stars? In Proceedings of the 17th Conference of Young Scientists and Specialists, Dubna, Russia, 8-12 April 2013; pp. 22-26. Available online: http:/ / xxx.lanl.gov/abs/1304.7758 (accessed on 30 April 2013).

5. Benic, S.; Blaschke, D.; Alvarez-Castillo, D.E.; Fischer, T.; Typel, S. A new quark-hadron hybrid equation of state for astrophysics-I. High-mass twin compact stars. Astron. Astrophys. 2015, 577, A40. [CrossRef]

6. Blaschke, D.; Alvarez-Castillo, D.E. High-mass twins \& resolution of the reconfinement, masquerade and hyperon puzzles of compact star interiors. AIP Conf. Proc. 2016, 1701, 020013.

7. Alvarez-Castillo, D.E.; Blaschke, D.B. High-mass twin stars with a multipolytrope equation of state. Phys. Rev. C 2017, 96, 045809. [CrossRef]

8. Paschalidis, V.; Yagi, K.; Alvarez-Castillo, D.; Blaschke, D.B.; Sedrakian, A. Implications from GW170817 and I-Love-Q relations for relativistic hybrid stars. Phys. Rev. D 2018, 97, 084038. [CrossRef]

9. Seidov, Z.F. The Stability of a Star with a Phase Change in General Relativity Theory. Soviet Astronomy 1971, $15,347$.

10. Blaschke, D.; Alvarez-Castillo, D.E.; Benic, S. Mass-radius constraints for compact stars and a critical endpoint. In Proceedings of the 8th International Workshop on Critical Point and Onset of De confinement, Napa, CA, USA, 11-15 March 2013.

11. Tolman, R.C. Static solutions of Einstein's field equations for spheres of fluid. Phys. Rev. 1939, 55, $364-373$. [CrossRef]

12. Oppenheimer, J.R.; Volkoff, G.M. On Massive neutron cores. Phys. Rev. 1939, 55, 374-381. [CrossRef]

13. Abbott, B.P.; Abbott, R.; Abbott, T.D.; Acernese, F.; Ackley, K.; Adams, C.; Adams, T.; Addesso, P.; Adhikari, R.X.; LIGO Scientific Collaboration and Virgo Collaboration; et al. GW170817: Observation of Gravitational Waves from a Binary Neutron Star Inspiral. Phys. Rev. Lett. 2017, 119, 161101. [CrossRef] [PubMed]

14. Annala, E.; Gorda, T.; Kurkela, A.; Vuorinen, A. Gravitational-wave constraints on the neutron-star-matter Equation of State. Phys. Rev. Lett. 2018, 120, 172703. [CrossRef] [PubMed]

15. Bauswein, A.; Just, O.; Janka, H.T.; Stergioulas, N. Neutron-star radius constraints from GW170817 and future detections. Astrophys. J. 2017, 850, L34. [CrossRef]

16. Rezzolla, L.; Most, E.R.; Weih, L.R. Using gravitational-wave observations and quasi-universal relations to constrain the maximum mass of neutron stars. Astrophys. J. 2018, 852. [CrossRef]

17. De, S.; Finstad, D.; Lattimer, J.M.; Brown, D.A.; Berger, E.; Biwer, C.M. Tidal Deformabilities and Radii of Neutron Stars from the Observation of GW170817. Phys. Rev. Lett. 2018, 121, 091102. [CrossRef] [PubMed]

18. Steiner, A.W.; Lattimer, J.M.; Brown, E.F. The Equation of State from Observed Masses and Radii of Neutron Stars. Astrophys. J. 2010, 722, 33-54. [CrossRef]

19. Raithel, C.A.; Özel, F.; Psaltis, D. From Neutron Star Observables to the Equation of State. II. Bayesian Inference of Equation of State Pressures. Astrophys. J. 2017, 844, 156. [CrossRef]

20. Salmi, T.; Nättilä, J.; Poutanen, J. Bayesian parameter constraints for neutron star masses and radii using X-ray timing observations of accretion-powered millisecond pulsars. Astron. Astrophys. 2018, 618, A161. [CrossRef] 
21. Margueron, J.; Hoffmann Casali, R.; Gulminelli, F. Equation of state for dense nucleonic matter from metamodeling. II. Predictions for neutron star properties. Phys. Rev. C 2018, 97, 025806. [CrossRef]

22. Blaschke, D.; Alvarez-Castillo, D.E.; Klähn, T. Universal Symmetry Energy Contribution to the Neutron Star Equation of State. 2016. Available online: http:/ /xxx.lanl.gov/abs/1604.08575 (accessed on 29 April 2016).

23. Alvarez-Castillo, D.E.; Blaschke, D.B.; Grunfeld, A.G.; Pagura, V.P. Third Family of Compact Stars within A Nonlocal Chiral Quark Model Equation of State. 2018. Available online: http:/ /xxx.lanl.gov/abs/1805. 04105v3 (accessed on 1 January 2019).

24. Most, E.R.; Weih, L.R.; Rezzolla, L.; Schaffner-Bielich, J. New constraints on radii and tidal deformabilities of neutron stars from GW170817. Phys. Rev. Letters 2018, 120, 261103. [CrossRef]

25. Christian, J.E.; Zacchi, A.; Schaffner-Bielich, J. Signals in the tidal deformability for phase transitions in compact stars with constraints from GW170817. Phys. Rev. D 2019, 99, 023009. [CrossRef]

26. Montana, G.; Tolos, L.; Hanauske, M.; Rezzolla, L. Constraining Twin Stars with GW170817. 2018. Available online: http:/ /xxx.lanl.gov/abs/1811.10929 (accessed on 28 November 2018).

27. Sieniawska, M.; Turczanski, W.; Bejger, M.; Zdunik, J.L. Tidal Deformability and Other Global Parameters of Compact Stars with Phase Transitions. 2018. Available online: http://xxx.lanl.gov/abs/1807.11581 (accessed on 27 July 2018).

28. Alvarez-Castillo, D.; Ayriyan, A.; Benic, S.; Blaschke, D.; Grigorian, H.; Typel, S. New class of hybrid EoS and Bayesian M-R data analysis. Eur. Phys. J. A 2016, 52, 69. [CrossRef]

29. Kolomeitsev, E.E.; Voskresensky, D.N. Relativistic mean-field models with effective hadron masses and coupling constants, and rho- condensation. Nucl. Phys. A 2005, 759, 373-413. [CrossRef]

30. Röpke, G.; Blaschke, D.; Schulz, H. Pauli Quenching Effects in a Simple String Model of Quark / Nuclear Matter. Phys. Rev. D 1986, 34, 3499. [CrossRef]

31. Kaltenborn, M.A.R.; Bastian, N.U.F.; Blaschke, D.B. Quark-nuclear hybrid star equation of state with excluded volume effects. Phys. Rev. D 2017, 96, 056024. [CrossRef]

32. Ayriyan, A.; Bastian, N.U.; Blaschke, D.; Grigorian, H.; Maslov, K.; Voskresensky, D.N. Robustness of third family solutions for hybrid stars against mixed phase effects. Phys. Rev. C 2018, 97, 045802. [CrossRef]

33. Ayriyan, A.; Grigorian, H. Model of the Phase Transition Mimicking the Pasta Phase in Cold and Dense Quark-Hadron Matter. EPJ Web Conf. 2018, 173, 03003. [CrossRef]

34. Abgaryan, V.; Alvarez-Castillo, D.; Ayriyan, A.; Blaschke, D.; Grigorian, H. Two Novel Approaches to the Hadron-Quark Mixed Phase in Compact Stars. Universe 2018, 4, 94. [CrossRef]

35. Maslov, K.A.; Kolomeitsev, E.E.; Voskresensky, D.N. Relativistic Mean-Field Models with Scaled Hadron Masses and Couplings: Hyperons and Maximum Neutron Star Mass. Nucl. Phys. A 2016, 950, 64-109. [CrossRef]

36. Maslov, K.; Yasutake, N.; Ayriyan, A.; Blaschke, D.; Grigorian, H.; Maruyama, T.; Tatsumi, T.; Voskresensky, D.N. Hybrid Equation of State with Pasta Phases and Third Family Of Compact Stars I: Pasta Phases and Effective Mixed Phase Model. 2018. Available online: http:/ /xxx.lanl.gov/abs/1812.11889 (accessed on 31 December 2018).

37. Hinderer, T.; Lackey, B.D.; Lang, R.N.; Read, J.S. Tidal deformability of neutron stars with realistic equations of state and their gravitational wave signatures in binary inspiral. Phys. Rev. D 2010, 81, 123016. [CrossRef]

38. Abbott, B.P.; LIGO Scientific Collaboration and Virgo Collaboration. GW170817: Measurements of neutron star radii and equation of state. Phys. Rev. Lett. 2018, 121, 161101. [CrossRef] [PubMed]

39. LIGO Scientific Collaboration and Virgo Collaboration. GW170817: Measurements of Neutron Star Radii and Equation of State. 2018. Available online: https:/ / dcc.ligo.org/LIGO-P1800115/public (accessed on 30 January 2019).

40. Antoniadis, J.; Freire, P.C.C.; Wex, N.; Tauris, T.M.; Lynch, R.S.; van Kerkwijk, M.H.; Kramer, M.; Bassa, C.; Dhillon, V.S.; et al. A Massive Pulsar in a Compact Relativistic Binary. Science 2013, 340, 1233232. [CrossRef] [PubMed]

(C) 2019 by the authors. Licensee MDPI, Basel, Switzerland. This article is an open access article distributed under the terms and conditions of the Creative Commons Attribution (CC BY) license (http:/ / creativecommons.org/licenses/by/4.0/). 